\title{
THE MANAGEMENT OF AN OUTBREAK OF LEGIONNAIRES' DISEASE IN A GEOGRAPHICALLY ISOLATED DISTRICT GENERAL HOSPITAL
}

\author{
J Williams, Consultant Anaesthetist \\ Furness General Hospital
}

\section{INTRODUCTION}

This is an account of an outbreak of Legionnaires' disease which occurred in Barrow-in-Furness during late July and early August 2002. The outbreak was not only characterized by more than 150 definite cases, but also by an almost overwhelming number of associated admissions with suspected disease. The response of the trust, hospital and community staff in all specialities has to be recognised as a prime factor in the lower than expected mortality of the outbreak.

On Friday 26th July, patient T was admitted to the Medical Admissions Unit (MAU) at Furness General Hospital (FGH). The presumptive diagnosis, supported by radiographic evidence, was of atypical community acquired pneumonia Although there had been an unusual number of similar cases over the preceding week, this patient's condition deteriorated so rapidly that she was transferred to the Intensive Care Unit (ICU). There, she required artificial ventilation (IPPV) and was treated for the associated symptoms of septicaemia. At this point the suspicions of physicians and our microbiologist had been aroused. A urinary antigen test for Legionnaire's disease on patient $\mathrm{T}$ was found to be positive as were further tests on inpatients over succeeding days. By Thursday 1st August the trust had been alerted that there was likely to be a local origin for the outbreak, and the appropriate Public Health Authorities informed. Fortunately, due to their rapid reaction and to the limited number of possible sources in the town of Barrow-in-Furness, any likely infected plant was taken out of action within hours, thus limiting the outbreak and defining the latest date and time of onset of the disease.

Legionnaire's disease was first identified as a pathological entity following an outbreak at the 1976 National Convention of the American Legion in Philadelphia. The outbreak in that case is said to have been traced to the air conditioning plant of a hotel at which many of the participants were staying, and out of the 221 disease victims 34 died in the succeeding days and weeks. The organism in that and in most major outbreaks is Legionella pneumophila, although the longbeachae strain has been implicated in sporadic cases. This latter organism is often isolated, unusually, from preparations of potting compost. The organism is widespread in the environment, both in soil and water, the natural reservoir being the freshwater amoeba. Infection in humans is usually by the ingestion or inhalation of heavily infected water or aerosols. In the modern, air-conditioned society, commercial watercooled as opposed to domestic refrigerant style units are likely to remain the most potent potential source of infection.
The illness is seen in two distinct clinical forms, Pontiac fever and Legionnaires' disease, although the likelihood of the former merely being an attenuated form of the latter seems not to be generally considered.

Pontiac fever is an acute, febrile, 'flu-like' illness. It tends to be self-limiting and there is no identifiable pneumonic phase.

Legionnaires' disease, which has an incubation period of between two and ten days, tends to develop with very similar symptoms of lassitude, fever and occasionally rigors followed by the development of a dry cough and increasing dyspnoea. A number of patients in this outbreak also reported disabling weakness to the point of collapse. These symptoms may be accompanied by headaches, vomiting and diarrhoea, patients frequently being admitted with concomitant dehydration and hyponatraemia. A patchy pneumonia of lobar or multi-lobar distribution is the most frequent finding as the disease progresses. The infection appears to favour the lower lobes in many cases. With appropriate antibiotic therapy this resolves over a period of four or five days, but patients often report a slow recovery from the more ill-defined symptoms of lassitude and general debility. Patients may also progress to fulminant pneumonia requiring respiratory support and the multi-organ failure associated with septicaemia.

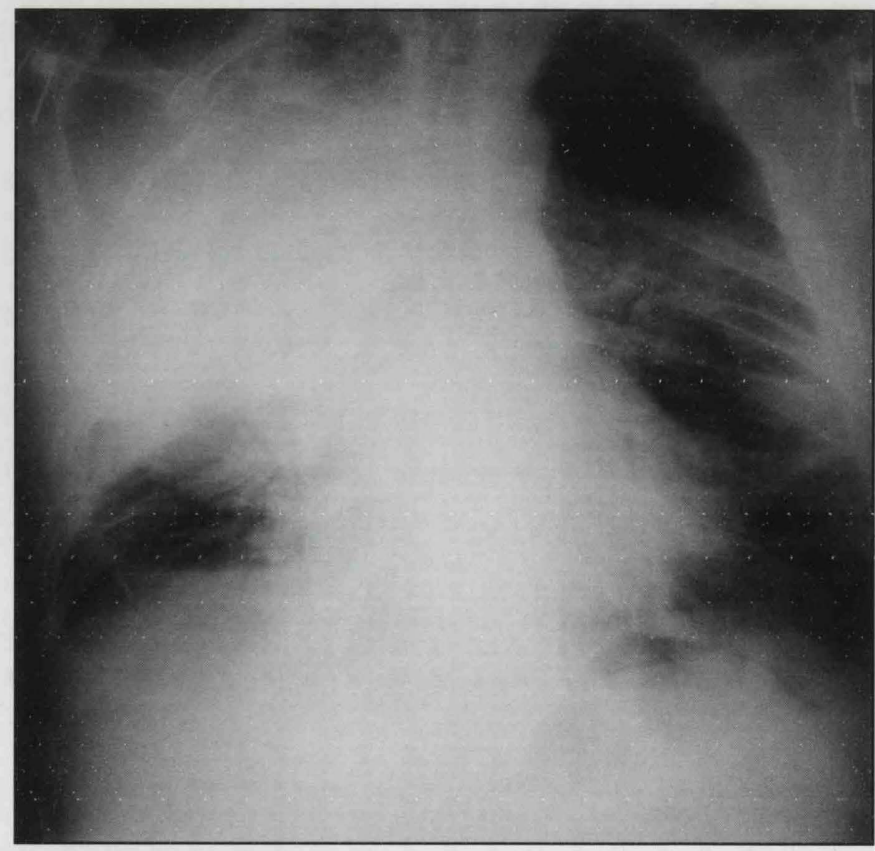

Figure 1 Legionnaires' pneumonia x-ray 


\section{MICROBIOLOGY}

Traditionally, the diagnosis of Legionnaires' disease has been made on the basis of the clinical features, supported by radiographic appearances and blood and sputum culture. A four-fold rise in antibody titre from acute and convalescent sera over a period of weeks rather than days is considered diagnostic but is uncommonly found and, in the case of this outbreak, has only been seen in one patient to date. By good fortune an immunological 'lollipop' style test had been recently introduced which detects the Legionella surface antigen excreted in the urine. The antigen, however, is not excreted by all patients or at all stages of the disease, positive tests being more commonly found in the early stages of the illness. Huge numbers of blood cultures were also taken in line with the British Thoracic Society guidelines for investigation of outbreaks of pneumonia, in an attempt to isolate a causal organism. Sputum culture in the case of Legionella strains is particularly difficult, the organism being fastidious in its requirements. Successful culture does enable very specific typing of the infecting strain to be made which may help to confirm the source of the infection.

To add to the general confusion, there had also been a number of cases of atypical pneumonia ascribed to a cyclical outbreak of Mycoplasma pneumoniae infections. With very similar symptoms and signs to the milder forms of Legionnaires' disease, this may help to account for some of the large number of referrals with symptoms of a chest infection, which also responded to the prescribed antibiotic regime.

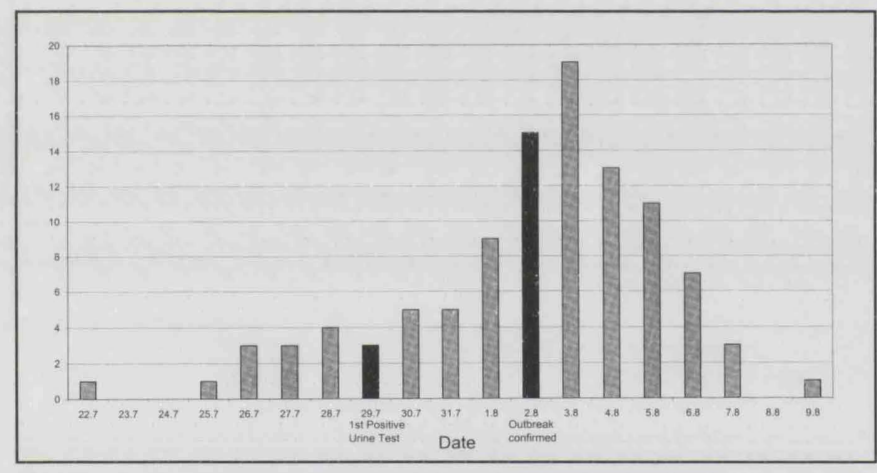

Figure 2 Positive antigen test demonstration

It can be seen that the workload for a relatively small microbiology department consisting of six WTE technical staff and one consultant would be likely to be overwhelming. During the outbreak, in excess of 2,400 urine tests were carried out by the staff at FGH, satisfying not only the needs of $\mathrm{A} \& \mathrm{E}$ and inpatients but also of an open access testing service for general practitioners (GPs). During the busiest period the advantages of close links with the laboratory at the Royal Lancaster Infirmary (RLI) enabled a large amount of work to be transferred there, and blood culture specimens were similarly sent to Preston. Urine specimens found to be positive or equivocal were also retested at the Central Public Health Laboratory, normal practice under these conditions. (Figure 2)

Following a meeting of clinicians, microbiologists, public health officers and executive staff on Thursday 1st August, a limited Major Accident Alert was put into action and the first of many press briefings arranged for the following morning. In keeping with the predicted seriousness of the outbreak all elective surgery was immediately cancelled to free up inpatient beds. This was to last for four weeks and to result in a backlog of some $600-700$ patients. A recently opened fiveday ward (13 beds) was made available for inpatient use as was the Day Case Unit (18 beds), appropriate staffing being achieved by the redeployment of ward staff, theatre staff and the liberal use of voluntary overtime. Major efforts were made to discharge as many pre-existing non-Legionella patients as possible within the bounds of safe clinical practice, which put an additional strain on overworked medical, clerical and pharmacy staff. In addition the two pitfalls of the outbreak occurring in both the peak annual leave season and at the changeover period for junior medical staff were making themselves felt. A call for locum medical staff was immediately made as was a call for help for more experienced staff from departments in the northwest region.

An 'incident room' was set up near to the A\&E department, manned by a management and medical team led by the chief executive. They were advised by a group of clinicians, and heads of all the major hospital departments, as well as by public and environmental health doctors, thus enabling an overview of the outbreak to be available at all times. Secretarial support was also of great importance here, collating data on admissions and responding to the huge number of incoming calls from an increasingly attentive press. The incident room rapidly became the central reference point for the outbreak, press releases being issued on a twicedaily basis. The releases were also distributed most importantly to hospital staff at all levels, as well as to GPs. Urgent problems as various as supplies of equipment, urine testing kits or transfer of patients were also promptly dealt with by the team, supplemented at an early stage by an ambulance liaison officer.

Simultaneously a helpline was set up, manned by medical, secretarial and other administrative staff. The huge public response to the news of the outbreak can be measured both by the attendances at the A\&E department and by the calls to the helpline (Figure 3). Fortunately this soon moderated and it was possible to scale down the team at the end of the first week of the outbreak

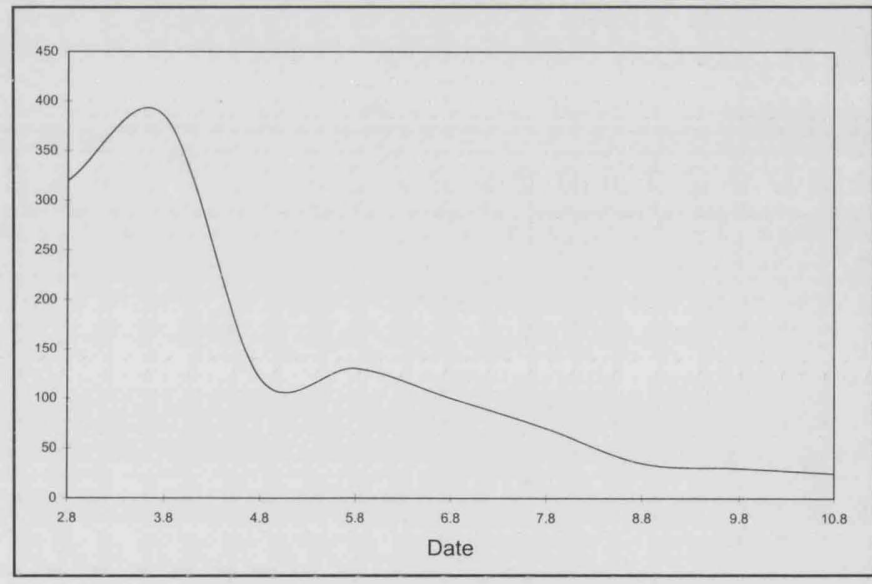

Figure 3 Calls to the helpline

All suspected Legionnaires' patients were triaged in the A\&E department according to a clerking proforma which was introduced and refined on almost a daily basis by the A\&E consultant. There were, as mentioned above, significant problems with inexperienced junior staff who nevertheless responded magnificently under very difficult circumstances, with the help of additional locum staff. A\&E nursing and clerical staff faced a mountainous task, especially on the first few days of the outbreak. Figure 4 indicates the peak of suspected admissions as occurring between the 3rd and the 6th August, A\&E records indicating a peak of 140 
attendances and more than 60 admissions in total (Figure 5). Anecdotally, staff reported a lower than average attendance by patients with minor complaints, but GPs reported an equivalent rise in consultation rates. Interestingly, a retrospective audit of $A \& E$ attendances of patients with selfpoisoning revealed an excess of cases when compared with the same period two years previously.

All patients seen with suspected Legionella were allocated to one of three categories if the decision was made to admit.

\section{Legionella clinically diagnosed or suspected}

\section{Legionella remains clinically possible}

\section{Legionella clinically excluded (state alternate diagnosis).}

If in the first two categories, all patients were prescribed appropriate antibiotics (see below), given oxygen, an iv line sited and given any other medication as appropriate; analgesics or anti-pyretics for example. Blood and urine samples were also taken at this point, many anxious patients happy to be discharged once the result of a urine test was found to be negative. For suspected cases, transfer to an inpatient bed was then made as rapidly as possible to free up $\mathrm{A} \& \mathrm{E}$ areas and a regular update on all suspected cases was passed on to the coordinating team in the incident room.

The chosen antibiotic regimes were Clarithromycin 500 $\mathrm{mg}$ iv given in $250 \mathrm{mls} \mathrm{N} / \mathrm{Saline}$ over one hour for the seriously ill who were unable to tolerate oral medication, with the addition of Rifampicin $300 \mathrm{mg}$ bd in the severely ill. For those sensitive to Clarithromycin, Ciprofloxacin was prescribed in a dose of $400 \mathrm{mg}$ bd intravenously or $500 \mathrm{mg}$ bd orally. After three to five days, in the event of good recovery, the iv regime was replaced by oral Erythromycin 500mg qds for ten days. No patients taking Rifampicin were discharged until they had finished the course of medication due to the potential hepatotoxic side effects. Due to the high demand for

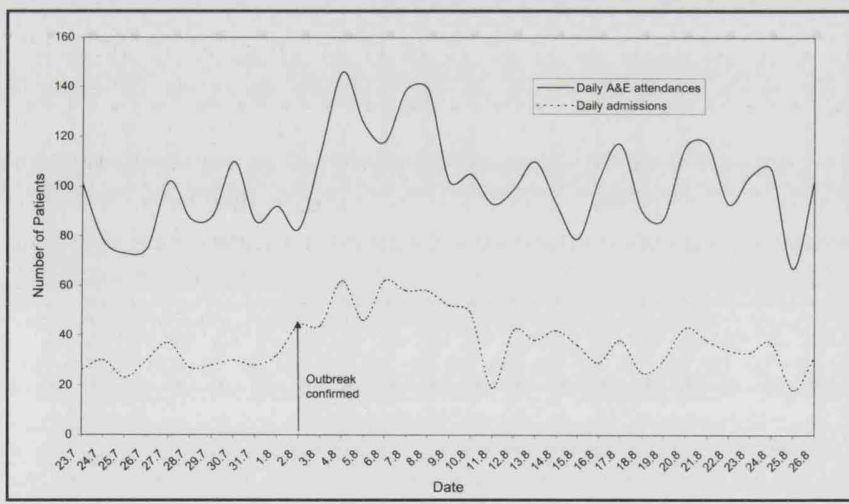

Figure 4 A\&E attendances compared with admissions

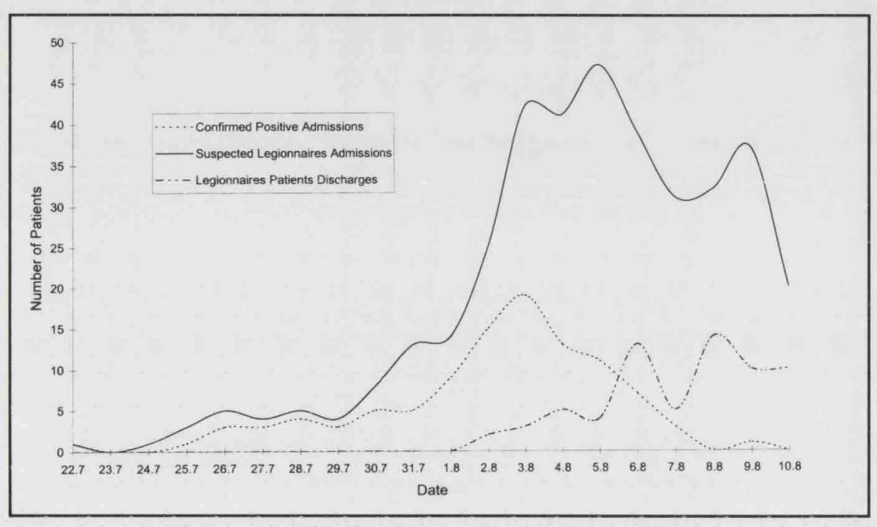

Figure 5 Legionnaires' cases confirmed vs suspected iv infusions it was decided to prepare Clarithromycin infusions in the pharmacy, although this added hugely to their workload. In addition there was an increasing demand for parenteral nutrition for the seriously ill, as well as routine work for the oncology unit, for example. Again, assistance was forthcoming from RLI and in ten days 1,500 bags of Clarithromycin were prepared, equivalent to the usage in an average year. In addition, dispensary workload increased by $25 \%$ resulting, over a ten-day period, in a total in excess of 300 additional hours of work performed by pharmacy staff.

Similarly the imaging department was under considerable strain due to the demand for chest x-rays. Initially the demand was underestimated, but as practically all admissions to A\&E with any signs or symptoms of Legionnaires' disease were referred for radiological examination the true situation was rapidly revealed. Radiographers immediately volunteered for additional shifts, as did clerical staff. A\&E nursing staff were allowed to request CXR under protocol and additional portering staff were deployed to facilitate the movement of patients. Support was also forthcoming from other sites within Morecambe Bay from radiography, radiology and clerical staff. The scale of the task is indicated in the accompanying graph (Figure 6) and has continued in the form of the weekend follow-up clinics.

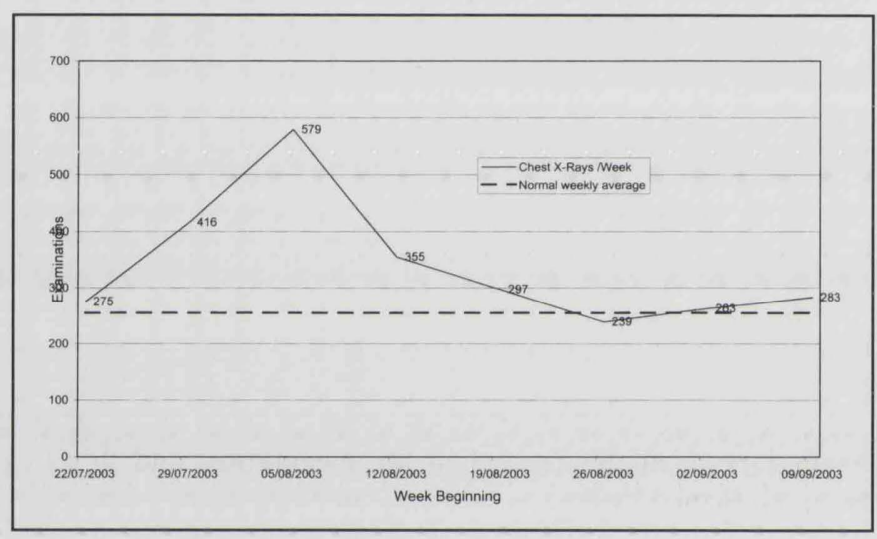

Figure 6 Chest $x$-rays per week

As numbers of admissions rose in a meteoric fashion over the first few days of August, it became apparent that certain patients were prone to a worsening of their condition over a time scale measured in hours. It was essential therefore to set up an early warning service, which commenced on the evening of Saturday 3rd August. This was manned by a succession of senior nursing staff, most of whom had intensive care training, as well as the hospital resuscitation officer. An early warning system scoring chart was rapidly formatted and distributed. The plan was to maintain fourhourly ward rounds concentrating on the patients giving most anxiety to ward staff. In the event of a significant deterioration, the opinion of an intensivist or consultant physician was obtained. If necessary the patient could then be transferred to ICU for observation or stabilisation. In the intervening periods medical and nursing ICU staff responded to reports on individual patients as necessary and due to the ease of communication in the smaller hospital were able to react rapidly to avert crises.

The six ICU beds available in FGH rapidly filled with severely ill patients, all of whom required ventilation and in most cases haemofiltration for renal failure. The problem then became deciding which patients to transfer and how to manage the transfers safely. Fortunately FGH has a small but dedicated team of staff grade anaesthetists, who, along with consultant anaesthetists not required immediately on site, 
were able to accompany all the severely ill patients. A number of patients known to be positive for Legionnaires' disease, but not critically ill, had already been transferred to RLI, and we were able to make further use of the ICU beds there as more of the patients became critically ill. In all, 13 patients were transferred directly from the wards. Six Legionella positive, ventilated patients were transferred from ICU to nearby hospitals, as were three non-Legionella ventilated patients. All transfers were made without incident, although monitor battery life gave some cause for concern due to the short turnaround time between transfers. The majority of transfers were made by paramedic ambulance, and the presence of an ambulance liaison officer in the incident room made this relatively simple to arrange. Use was made of the Lancashire Air Ambulance, but this was somewhat limited by the time of day of transfers, the rather poor weather conditions and limits set on pilot flying hours.

As the first full week of the outbreak drew to a close it was clear that the peak had been reached and passed. Fortunately the apparent source of the infection was shut off on the day before the outbreak was made public. Thus there should not have been any further cases developing after the 11th August, and this proved to be the case. Many very poorly patients remained in hospital, however, and there had been two inhospital deaths. Discharge was deferred until the medical staff were completely satisfied with the clinical condition, and it was to be a further three weeks before normal service was resumed.

To date there have been positive tests on 155 patients and five definite and two possible deaths linked to Legionnaires' disease, the majority of the deaths occurring after an extended period of intensive care. This indicates a mortality rate in the region of $5 \%$, by far the lowest of any recent outbreak. The reasons for this are not at all clear and further research is in hand to elucidate the likely factors. However there is no doubt that the management of the outbreak was exemplary at all levels and in all specialities, and this has been confirmed by an external assessment.

\section{SOME FACTORS RELATING TO A LOWER THAN EXPECTED MORTALITY RATE}

1 Early recognition of the outbreak and institution of appropriate antibiotic regimes

2 Early warning system commenced on Sat 3rd August, alertness of nursing staff and ease of communication

3 Institution of a clerking proforma and admission of all suspected cases even though the numbers were very high

4 Assessment of all deteriorating cases by an ICU specialist or consultant physician

5 Early transport of critical cases to the nearest ICU bed

6 Exemplary support by the trust management, public health staff and the medical director, relieving clinicians of the administrative and public relations burden

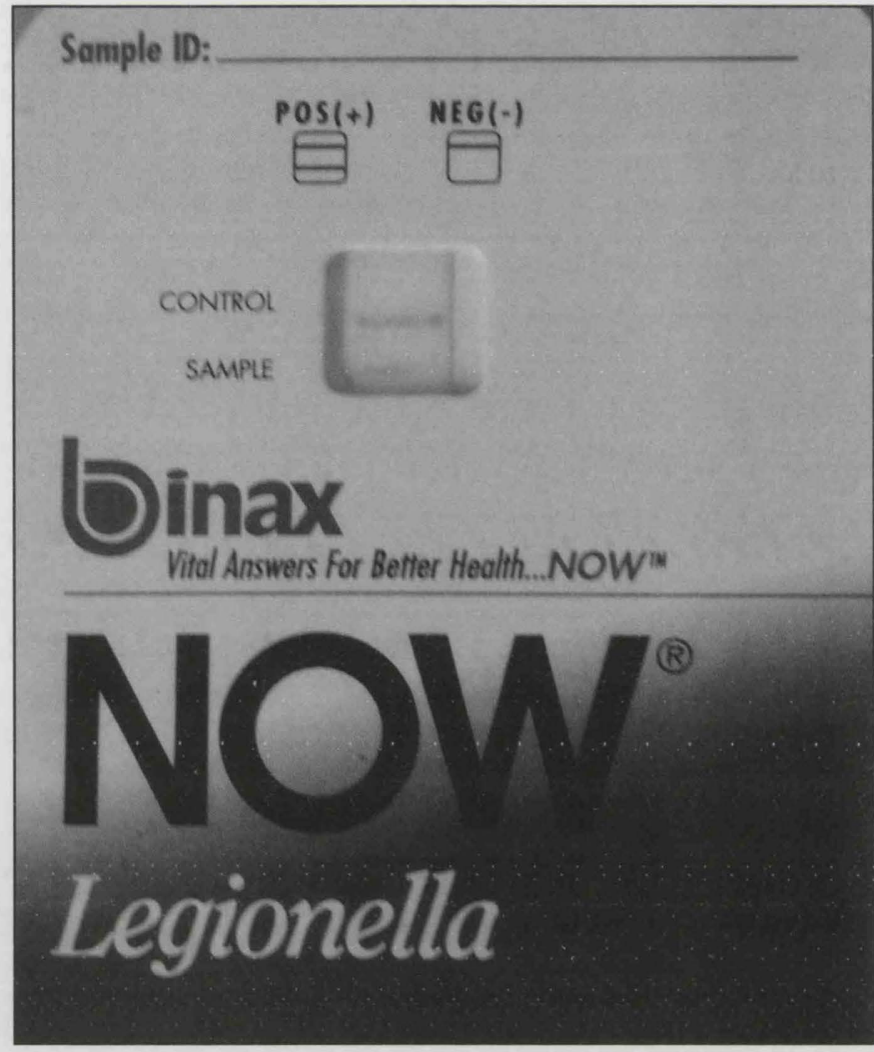

Figure 7 Urinary antigen test kit showing positive result

7 Unstinting support by nursing, clerical and domestic staff

8 GPs asked to follow strict guidelines and treatment protocols for any suspected case

9 The combined resources of two DGHs working as a single unit despite their geographical separation.

\section{POST SCRIPT}

As the results of follow-up clinics start to become available, the number of confirmed cases continues to grow. The count now stands at 155 , but in addition there are a further 38 patients who have shown a positive urine test but no serological changes. Until all the results are finalised it is difficult to apply a statistical analysis to them, but it would appear that the percentage of patients treated in ICUs is similar to those in other outbreaks, thus confirming the virulence of the organism. There have been no further Legionnaires'-related deaths.

\section{Acknowledgements}

I would like to express my thanks to all the colleagues who have so freely given of their time and provided much of the information and data recorded here. Also to Morecambe Bay Hospitals NHS Trust for permission to reproduce some of the epidemiological data. 